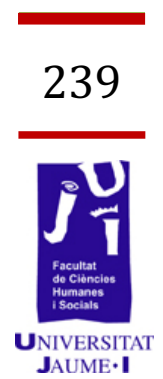

\title{
La educación plástica y visual en espacios de arte contemporáneo
}

Oihana Tárrega Astigarraga

al188543@uji.es

Paloma Palau Pellicer ppalau@uji.es 
El presente proyecto se enmarca en el apartado de trabajos prácticos vinculados al practicum con alumnado de primer ciclo de educación primaria. La experiencia llevada a cabo fue una visita escolar a un espacio de arte contemporáneo en la que se analizó la implicación de la institución y de la escuela, así como el interés del alumnado. Los objetivos de la investigación son la necesidad de acercar el arte contemporáneo al alumnado y establecer una relación educativa entre la escuela y el museo. El contexto para trabajar el arte contemporáneo fue el EACC (Espai d'Art Contemporani de Castelló) que permitió acercar el arte a los más pequeños de forma que lo relacionasen con algo atractivo. La propuesta consistió en una fase inicial de conocimientos previos antes de la visita, partiendo de conceptos básicos como son: pintura, escultura y museo. Además experimentaron los roles de comisario, artista, visitante y vigilante del museo. Después de la visita trabajamos actividades relacionadas con una obra titulada «Time Bank« de la exposición temporal «7.000.000.000» del EACC. Los resultados obtenidos con esta experiencia, sirvieron para que los alumnos/as apreciaran el arte, fueran críticos y disfrutaran con y de las manifestaciones artísticas. Además, les enseñamos a valorar y respetar el patrimonio cultural, comportarse en un espacio diferente y desarrollar su capacidad creativa con la visualización del arte contemporáneo, que no suele ser trabajado habitualmente en el aula.

Palabras clave: Educación Primaria, expresión plástica, arte contemporáneo, escuela, didáctica.

\section{Introducción}

La primera aproximación a la realidad de la educación en artes visuales en el aula fue durante el practicum del curso pasado. Fui consciente de que el arte no está presente en las aulas, a lo sumo se tratan artistas muy conocidos de la ciudad y se citan algunos otros para representar obras al final del curso. Las manualidades y el dibujo libre son los ejercicios habituales. Fue entonces cuando se instaló en mi la motivación por descubrir, investigar y trabajar en este campo, en el que, tanta falta hace elaborar propuestas acordes con la realidad de esta disciplina. Además del importante estímulo que han supuesto las asignaturas en artes visuales de la Universidad. Estas circunstancias han sido el fundamento para elaborar este trabajo vinculado con el practicum. Por otro lado, considero que es una situación oportuna para trabajar contenidos contemporáneos y metodologías acordes con las necesidades del alumnado.

En general, la mayoría de trabajos de investigación que existen sobre educación artística en contextos expositivos hacen referencia a lo que estos espacios ofrecen. Algunos museos españoles como el Thyssen, el Museo del Prado o el Guggenheim ofrecen en sus páginas web la posibilidad de orientar a los docentes antes de la exposición y proporcionarles fichas de trabajo previo a la visita. Olga Fernández en su 
trabajo comenta que "[...] las áreas educativas en los museos se encuentran en un momento de crecimiento y sistematización teórica e institucional» (Fernández, 2007: 19-24) y considera que uno de los objetivos es «[...] intentar paliar la falta de apreciación y el descrédito del arte contemporáneo y aumentar el disfrute y comprensión de las artes y el museo, fomentar un posicionamiento crítico, incluido el propio arte, desde el área de educación [...]» (Fernández, 2007: 19-24). Si bien es cierto, esta relación entre escuela y museo, no es nueva, ya que como señala Storksdieck «Desde la década de los 90 , se sabe de una aparente ganancia cognitiva de la visita al museo - contra el tratamiento del tema en el aula» (Storksdieck, 2006). En una investigación de María del Carmen Sánchez Mora para la Universidad Nacional Autónoma de México, sobre la relación museo-escuela y el trabajo realizado durante treinta años de investigación educativa, comprobamos que los diferentes autores citados, no contradicen lo anteriormente comentado. La autora defiende que «Una relación educativa realmente productiva entre el museo y la escuela conlleva que cada institución asuma sus potencialidades» (Sánchez Mora, 2013: 15-21). Además, se apoya en otros autores, como K. B. Lucas que comenta "Para tener una relación adecuada con estos grupos, requiere diseñar estrategias que tengan en cuenta como se aprende individual y grupalmente, como piensan los estudiantes de diferentes edades y como enseñan los maestros» (Lucas, 2000: 524-544). Si bien, se ha comentado el papel del museo, ahora hablamos del de la escuela, no menos importante, y es que según Camareno "[...] la visita constituye una herramienta de aprendizaje. En efecto, se ha encontrado que los maestros la utilizan para motivar a sus estudiantes, para enseñarles temas que, por alguna razón, no pueden cubrirse eficazmente en el aula, para complementar la enseñanza de otros e incluso, para acercar a los estudiantes a su propia comunidad» (Camareno, 2009: 151-165).

En cuanto a lo referido a la enseñanza del arte en las escuelas, autores de referencia como Elliot W. Eisner que justifica la necesidad de enseñanza del arte de manera "contextualista y esencialista» (Eisner, 1995: 2). O para la redefinición del concepto, como la autora María Acaso «[...] el arte contemporáneo nos puede aportar mucho[...] hay que cambiar el paradigma de la educación artística como manualidad a la educación artística como vehículo de conocimiento [...]» (Acaso, 2009: 112). El futuro no es demasiado esperanzador, ya que, actualmente la Ley educativa LOMCE (2013) distingue entre materias troncales, específicas y de especialización. La plástica se encuentra entre las específicas, eso quiere decir que será una materia optativa y serán las comunidades autónomas y los colegios los que determinen si se ofrecerá o no y el tiempo que se le dedicará. Comprobamos pues, que en contra de todas las opiniones mostradas por los anteriores autores citados, se va a producir un retroceso y una paulatina disminución de esta asignatura en mi opinión tan importante en la programación escolar. 


\section{Objetivos}

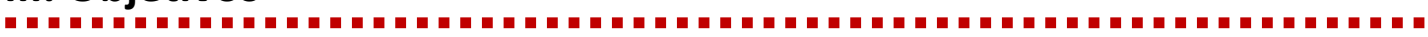

\subsection{Antecedentes}

Antes de comenzar considero importante contextualizar la materia en la que vamos a desarrollar nuestro proyecto. Partimos de que la Educación Artística en la escuela abarca la educación musical y plástica. Aunque bien es cierto que, en términos generales, comparten todo lo relacionado con el proceso creativo, cada una tiene su propio lenguaje. Esta circunstancia, sin embargo, provoca que en la gran mayoría de los centros a la educación plástica se le destine menos tiempo dentro del horario escolar pero lo peor de todo, es que el poco que se le dedica es para hacer manualidades y no se trabaja el arte. Un debate actual entre los especialistas en la materia que se resisten a estás prácticas sitúan el origen en la formación del profesorado. Pero no sólo esto preocupa, después de una investigación por la red, podemos encontrar multitud de recursos para educación primaria relacionados con el Arte Clásico. Algunos colegios en sus páginas web cuelgan trabajos realizados por sus alumnos sobre autores como Velazquez, Goya, Sorolla y otros de épocas más modernas, como Miró, Dalí y Picasso. Por no hablar de las editoriales que, el curso pasado en la asignatura de Didáctica de las Artes Visuales, tuvimos oportunidad de analizar. El material que ofrecían es desolador, algunas incorporan libros con unidades didácticas en las que la experimentación y creatividad es escasa e incluso nula como podemos apreciar en el recurso educativo «Diego Velazquez» (Recursosep, 2010).

En otras editoriales nos ofrecen un maletín de recursos, con todos los materiales necesarios según ellos para plástica, es decir, volvemos a las manualidades, como podemos ver en el «Programa Tradid 4엉 de Primaria» (Tradid, 2013).

Sin embargo, no todo lo que nos encontramos es negativo, también tenemos noticias sobre centros donde se realizan actividades que acercan el arte contemporáneo al alumnado, como esta aparecida en el Diario de Navarra en 2014 «Alumnos de primaria se acercan al arte contemporáneo en Huarte». En otra noticia encontramos como la Mustang Art Gallery desarrolla el proyecto didáctico «MAG Satelite" para acercar la creación artística al alumnado de bachillerato, «El arte contemporáneo irrumpe en las aulas» (MAG, 2013). «La experiencia está resultando muy positiva ya que nos está sirviendo para llevar el arte hasta las mismas aulas, motivando a los chicos y chicas y haciéndoles directamente partícipes de esta actividad artística que sin duda está dando ya sus primeros frutos incentivando y despertando el espíritu creativo de muchos de los alumnos" según comenta Juan Fuster director del espacio MAG. De modo que también hay iniciativas relacionadas con la escuela y el arte contemporáneo aunque más común en las aulas de secundaria y bachillerato, pero no tanto en educación primaria. Para que ello ocurra, tendríamos que tener presentes a especialistas en la materia como Guillermo Machuca y Alicia Villarreal que comentan: 
Es tarea de todos los profesionales relacionados con la red de las artes visuales, especialmente de aquellos provenientes del mundo de la educación, entregar herramientas que permitan a los jóvenes reconocer y recrear las claves proyectadas por el arte contemporáneo. Esto implica desarrollar instancias educativas innovadoras que contribuyan a mejorar la capacidad de descifrar las obras de arte, así como a integrarse a un proceso de apropiación e interpelación del medio visual, urbano y comunicacional cada vez más cambiante y complejo. (Machuca y Villarreal, 2012)

Analizados los antecedentes en el estudio de la Educación plástica en primaria y concretamente en lo que al arte contemporáneo se refiere, coincidimos con la afirmación de Ricardo Marín-Viadel que considera «[...]urgencia social los procesos de aprendizaje y enseñanza de las artes visuales en la escuela[...] en instituciones tales como los museos» (MarínViadel, 2003: 448). Es por esto, la elaboración de este recurso que incorpora una salida a un espacio de arte contemporáneo con una trabajo previo y posterior a ella.

\subsection{Marco de referencia}

La relación entre la educación plástica museo-escuela y espacios de arte está cobrando cada vez más importancia. Para abrir sus colecciones o exposiciones a la sociedad los gabinetes pedagógicos se preocupan de generar actividades didácticas para atraer a las escuelas. Si bien es cierto que en todas ellas, el arte contemporáneo todavía no está demasiado extendido, sobretodo en las primeras etapas escolares. Esta circunstancia ha sido fundamental para llevar a cabo esta experiencia y que se recoja en un recurso didáctico. El trabajo realizado se adscribe a los argumentos expuestos anteriormente por especialistas en el campo de la educación en las artes visuales y las escuelas. Entre ellos, Elliot W. Eisner que en su libro Educar la visión artística justifica la necesidad de enseñar arte, como comentábamos anteriormente, por un lado de un modo contextualista y por otro esencialista. Para el primero, afirma que "[...]subraya las consecuencias instrumentales del arte en el trabajo y utiliza las necesidades concretas de los estudiantes o de la sociedad como base principal con la que conformar sus objetivos» (Eisner, 1995: 4). A si mismo, del tipo esencialista remarca que "[...] el tipo de contribución a la experiencia y al conocimiento humanos que sólo el arte puede ofrecer; acentúa lo que el arte tiene de propio y único» (Eisner, 1995:3).

El autor defiende la enseñanza del arte en su justificación contextualista argumentando que sólo se puede determinar correctamente un programa educativo si se comprende el contexto en el que dicho programa se va a aplicar, por lo que es necesario considerar las características de los estudiantes así como las necesidades de la sociedad. En contrapartida a esta justificación, encontramos a los esencialistas que defienden que la educación del arte no debería depender de quién vaya a recibir dicha educación, sino que el arte debe aportar sus valores implícitos y sus características específicas. El arte debe ofrecer a la educación del 
hombre lo que otros ámbitos no pueden, una experiencia que vivifique la vida.

Si profundizamos en las diferentes justificaciones, no todos los teóricos sobre la educación de arte coinciden en sus conclusiones y reclaman conjuntos diferentes de valores, entienden que los programas de las escuelas deben diferir para adaptarse a las necesidades del niño/a. Por un lado Viktor Lowenfeld defiende que "Si los niños se pudieran desenvolverse sin ninguna interferencia del mundo exterior, no sería necesario proporcionarles estimulo alguno para su trabajo creador» (Lowenfeld, 1980: 20). Por el contrario Irving Kaufman afirma que «[...]el contenido de la educación de arte ha sido ambivalente y vago, con frecuencia apartado de las amplias condiciones que conforman la naturaleza del arte» (Kaufman, 1965: 25). Presentadas las dos posiciones, queda claro la diferencia entre la contextualista que busca lograr los objetivos previamente estipulados en el programa, y la esencialista que defiende que la educación debería variar dependiendo de quien la fuera a recibir. Otro importante autor en la enseñanza del arte es John Dewey que sostiene que el arte vivifica, aporta sentimientos y experiencias a cada uno de nosotros. Resumiendo, con todo lo anteriormente expuesto llegamos a diferenciar las diversas funciones que la enseñanza del arte aporta a las personas. La principal función a destacar será la pedagógica, fundamental para el crecimiento del alumnado en cuanto a sus reflexiones y experiencias. La segunda función será emocional, basada en la sensibilidad que aporta el arte a cada individuo. Y por último la función crítica en cuanto a que el arte es un medio utilizado por el hombre para reflejar sus valores y la situación del mundo que lo rodea.

Valorado ya todo lo relativo a la necesidad de enseñar arte y a su justificación, y concretando en como se produce el aprendizaje, volvemos a centrar nuestra atención en el pensamiento desarrollado por Eisner en su libro anteriormente mencionado, y especialmente en su cuarto capítulo. El autor resalta que no hay un único camino para el aprendizaje artístico y que no es automático con la madurez, sino que es un proceso de evolución continua y diferente en cada individuo. Es por esto, por lo que dependerá de tres grandes aspectos a tener en cuenta: productivo, crítico y cultural. Si nos centramos en el primero de ellos, el aspecto productivo, es decir, como se aprende por ejemplo a dibujar o a esculpir, el autor señala cuatro factores que afectan a este aprendizaje:

- Habilidad en el tratamiento del material.

- Habilidad en la percepción de las relaciones cualitativas entre las formas producidas en la propia obra, entre las formas observadas como imágenes mentales.

- Habilidad de inventar formas que satisfagan a quien las realiza, dentro de los límites del material con el cual está trabajando.

- Habilidad en la creación de orden espacial, orden estético y capacidad expresiva.

En segundo lugar, en lo relativo al aspecto crítico, resulta de vital importancia el desarrollo de las capacidades perceptivas de cada individuo. 
Eisner expone seis tipos diferentes de dimensiones que intervienen en este proceso que son:

- Dimensión experiencial, el niño transmite lo que le hace sentir la obra.

- Dimensión formal, el observador busca las relaciones entre los elementos de la obra.

- Dimensión simbólica, la obra de arte utiliza símbolos que el observador tendrá que interpretar.

- Dimensión temática, el significado subyacente que el artista da a su obra.

- Dimensión material, elección de los materiales a utilizar en la obra.

- Dimensión contextual, la obra de arte como parte de un todo en la historia artística.

Por último y para terminar, encontramos el aspecto cultural, que el autor relaciona con la capacidad de comprender el arte como un fenómeno cultural de la sociedad. Para ello y teniendo en cuenta que la capacidad de percibir las relaciones de la obra con el medio, se desarrollan progresivamente con el aprendizaje del sujeto, Eisner señala la influencia de tres mecanismos cognitivos:

- Constancias visuales. Son interferencias entre lo que vemos y lo que sabemos mediante generalizaciones concretas que hemos desarrollado durante nuestro aprendizaje.

- Estructuras de referencia. Debido a las percepciones anteriores en nuestro aprendizaje, tendemos a generalidades y no particularidades sobre los objetos.

- Proceso de centralización. Se refiere al proceso en el que el individuo se centra en un aspecto concreto del objeto y pasa por alto la relación del mismo con el medio.

\subsection{Objetivos del estudio}

El objetivo principal es acercar el arte contemporáneo al aula con una visita a un espacio de arte, en este caso el Espai d'Art Contemporàni de Castelló (EACC), buscando que los niños/as interactúen con el medio natural, social y cultural que nos rodea, que conozcan la existencia de este tipo de lugares y que disfruten de ellos. Para ello se ha diseñado un recurso didáctico de cinco semanas de duración que comenzó con un trabajo previo en el aula. Además, con la visita al citado espacio se pretende fomentar las relaciones entre colegio y EACC, provocando la necesidad de trabajar en equipo para crear un recurso didáctico adaptado al nivel de los alumnos/as, generando conocimiento, acceso, formación e interés por comprender el arte contemporáneo en sus diversas manifestaciones y favorecer la comunicación de las artes plásticas. 
Con todo ello se busca logar en el alumnado una actitud positiva frente a las artes, desarrollando mediante los sentidos las competencias emocionales, educando la sensibilidad artística a través de valores sociales como la interculturalidad y el medioambiente entre otros.

\section{Material y metodología}

\subsection{Contexto institucional y espacio-temporal}

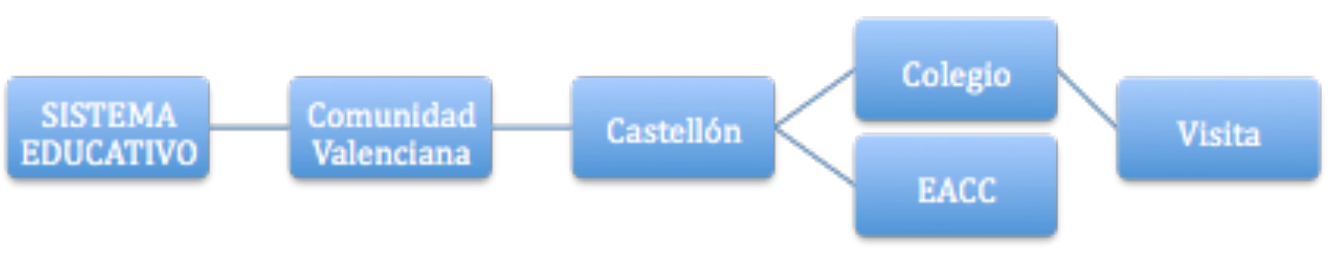

Figura 1. Esquema conceptual del contexto y las relaciones entre las instituciones.

Como podemos apreciar en el esquema, partimos del Sistema Educativo Español que es el que marca la estructura que debe seguir todo el país según lo establecido por el Ministerio de Educación. El siguiente escaño lo ocupa la Comunidad Valencia, que se manifiesta en el Decreto $(111 / 2007)$ indicando las particularidades de nuestra comunidad. Continuamos con la ciudad en la que nos encontramos y los lugares que forman parte de este proyecto de investigación con una duración de tres meses, que son el Colegio, por ser el lugar donde realizo las prácticas y el Espacio de Arte Contemporáneo de Castellón que es el lugar donde se desarrolla parte de mi trabajo.

Dentro del colegio, contamos con el equipo directivo, docentes, comunidad educativa y niños/as, que entre todos hacen posible que esta experiencia se lleve a cabo. A su vez, dentro del EACC incluimos al personal que allí trabaja y a las instituciones que lo subvencionan.

\subsection{Intervención/material}

\subsubsection{Objetivos del programa/material}

La experiencia realizada en el trabajo final de grado me ha servido para elaborar un material didáctico y a su vez trabajarlo con los alumnos/as en el aula. Los objetivos principales son: en primer lugar, acercar el arte a través de una salida escolar y trabajar el currículum, concretamente el área de educación plástica conjuntamente. Así como también favorecer el interés por la cultura, que aprecien y conozcan diferentes manifestaciones artísticas, que experimenten la manipulación de diversos materiales visuales y manuales, generar aprendizajes, curiosidad, interés, experimentar nuevas sensaciones y que desarrollen el sentido del gusto y capacidad crítica. 


\subsubsection{Participantes}

Los participantes serán el alumnado de primer ciclo de Educación Primaria, de edades comprendidas entre los 6 y los 8 años. Dentro del mismo ciclo, el colegio determina que en ambos cursos (primero A/ B y segundo $A / B$ ) se realicen las mismas actividades, por lo que el recurso didáctico se ha ejecutado de igual forma. En lo que respecta al número de alumnos/as tanto en primero como en segundo no varía demasiado de unas clases a otras siento el total 25 y el mínimo 23.

Si entramos a detallar las características principales del alumnado, existe un predominio de niños por encima de niñas. Pese a tener la misma edad, ambos cursos son diferentes, puesto que en primero los alumnos/as preferían áreas como conocimiento del medio o matemáticas y en segundo se decantaban por otras como educación plástica o educación física. Este detalle pude comprobarlo con las calificaciones, lo que me llevó a pensar que en general unos prefieren áreas técnicas y otros artísticas. Estos aspectos son fundamentales para llevar a cabo el recurso didáctico y con ello el fomento del gusto por las artes. En ambos casos, tras saber el contenido de las actividades manifestaron mucho entusiasmo e interés por hacer $y$ aprender nuevas actividades.

\subsubsection{Contenido a trabajar}

El contenido a trabajar en este proyecto corresponde al Bloque 1, centrado en la observación plástica, y el Bloque 2 dedicado a la expresión y creación, extraídos del Decreto 111/2007 de la Comunidad Valenciana.

El primer bloque, se centra principalmente en el análisis, comentario y observación de obras plásticas, agudizando el sentido de la vista, e interpretando todo aquello que es percibido. El segundo se ocupa de la experimentación manipulativa de todo tipo de objetos hasta llegar a la creación plástica propia, además de desarrollar una actitud crítica frente a las manifestaciones artísticas.

\subsubsection{Procedimiento}

El recurso didáctico que a continuación se presenta, titulado "Arte Dentro y Fuera de la Escuela» comprende una serie de actividades con una duración total de un mes, estructurado en 4 semanas y tres bloques diferenciados: pre-visita, visita y post-visita. Expuesto de manera detallada en el apartado de desarrollo de las sesiones.

Pre-visita: Una semana antes de la salida, en el aula mostramos al alumnado una serie de obras de arte de diferentes estilos, técnicas y artistas tanto nacionales como internacionales. Con ello conseguimos recabar información de sus conocimientos sobre el tema a tratar, trabajamos el comportamiento en otros espacios diferentes, los tipos de museos, lo que podemos encontrar en ellos, la gente que allí trabaja, etc. De igual modo tratamos también la experimentación de los diferentes roles 
como seguridad, comisario, artista o visitante. Después continuamos con la salida a la exposición y ya de vuelta al aula, en las dos semanas siguientes terminamos con actividades relacionadas con la salida. De esta manera, todo tenía un sentido para los alumnos/as y el aprendizaje fue más significativo.
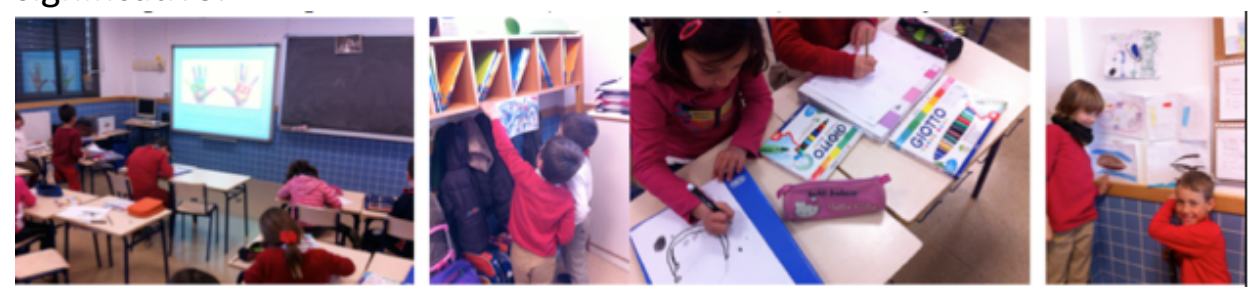

Figura 2. "Visitante y Artista" Montaje fotográfico de Oihana Tárrega.

Visita: La segunda semana realizamos la salida al EACC, con ello se pretendía que los alumnos/as fueran capaces de ver más allá en lo que al arte se refiere, que aprendieran con él y no de el. No buscamos que sean especialistas en arte contemporáneo, sino que desde una edad temprana, en mi caso los 6-7 años de edad conozcan la cultura de su tiempo, que observen y comprueben por ellos mismos la realidad que existe. Para lograrlo, pusimos a nuestros alumnos/as en contacto directo con el arte, aprendiendo de una manera activa, amena, participativa y principalmente significativa. Así pues, el objetivo de la salida fue una experiencia nueva, en un contexto diferente en el que adquirir conocimientos y aprender a comportase en otras situaciones diferentes al aula.

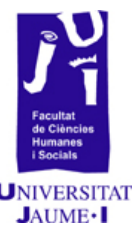



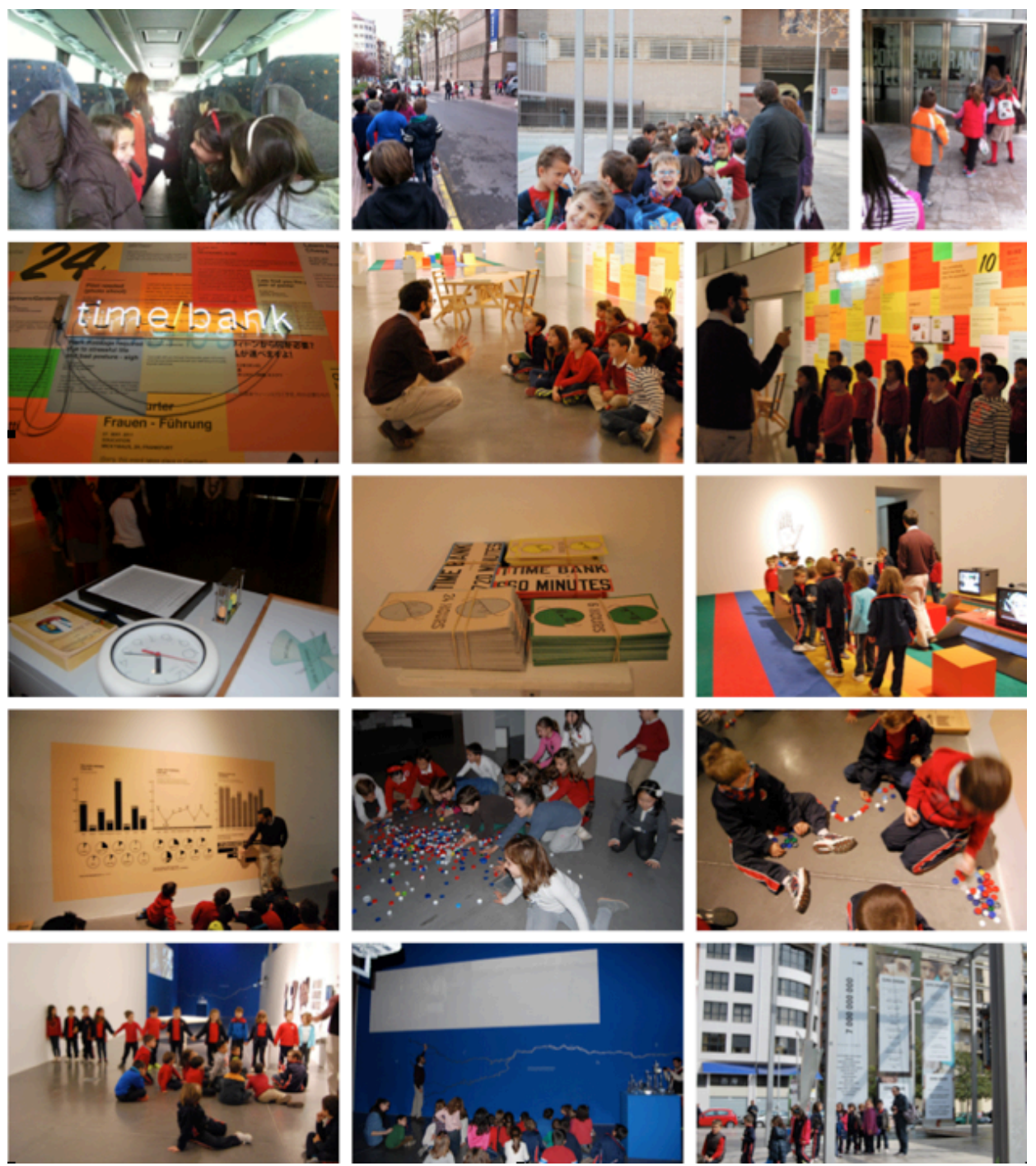

Figura 3. "Salimos del cole" Montaje fotográfico de Oihana Tárrega.

Post-visita: Las dos semanas siguientes, trabajamos aquello que vimos en la exposición. Junto con el comisario, se seleccionó la obra más interesante para trabajar con los alumnos/as según sus características y necesidades. En el aula recordamos de manera visual lo vivido y nos centramos específicamente en la obra titulada «Time Bank», que nosotros posteriormente llamamos «Banco de Aprendizaje» y sobre ella realizamos varias actividades adaptadas al aula. La primera actividad, recopilación de información sobre los conceptos, la segunda la elaboración y estampado de sellos para diseñar los carteles con las ofertas y demandas, la tercera el diseño de billetes de intercambio y por último llevamos a cabo la experiencia. En el aula montamos nuestro propio «Banco de Aprendizaje», cuyo objetivo consistía en la ayuda de unos a otros para que intercambiasen conocimientos, habilidades o destrezas. 
FÒRUM DE RECERCA - ISSN 1139-5486 - http://dx.doi.org/10.6035/ForumRecerca.2014.19.17 №19/2014. pp. 239-258
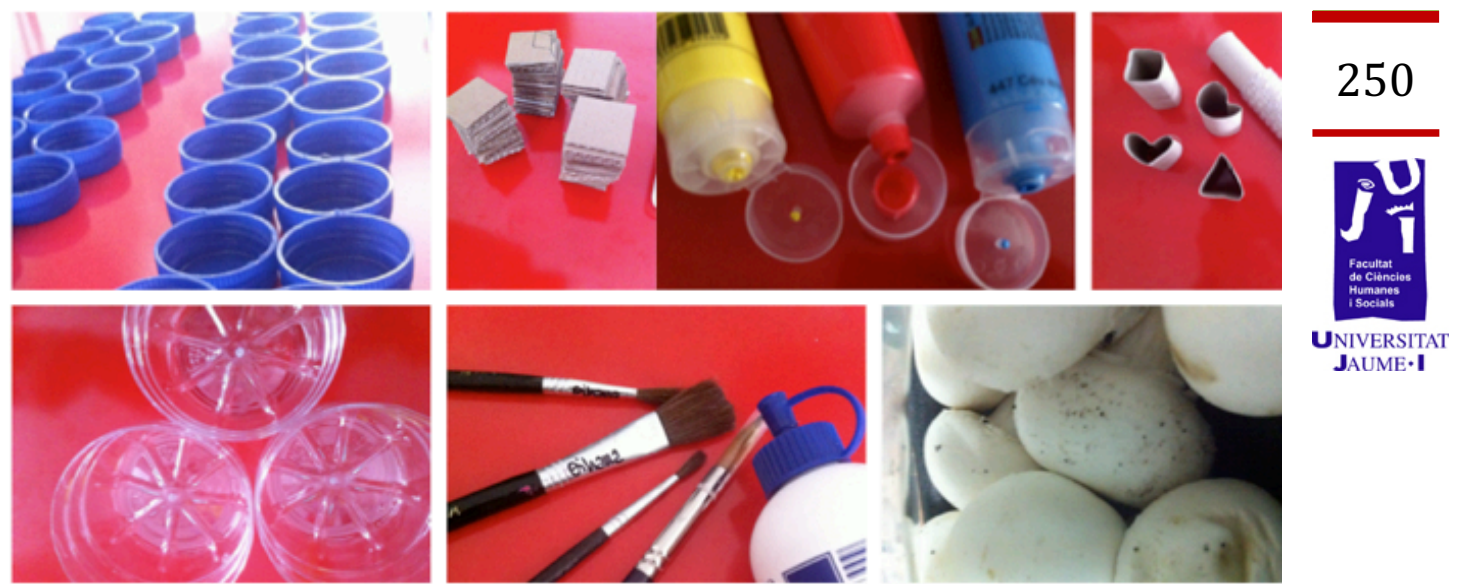

JAUME $\mathbf{I}$
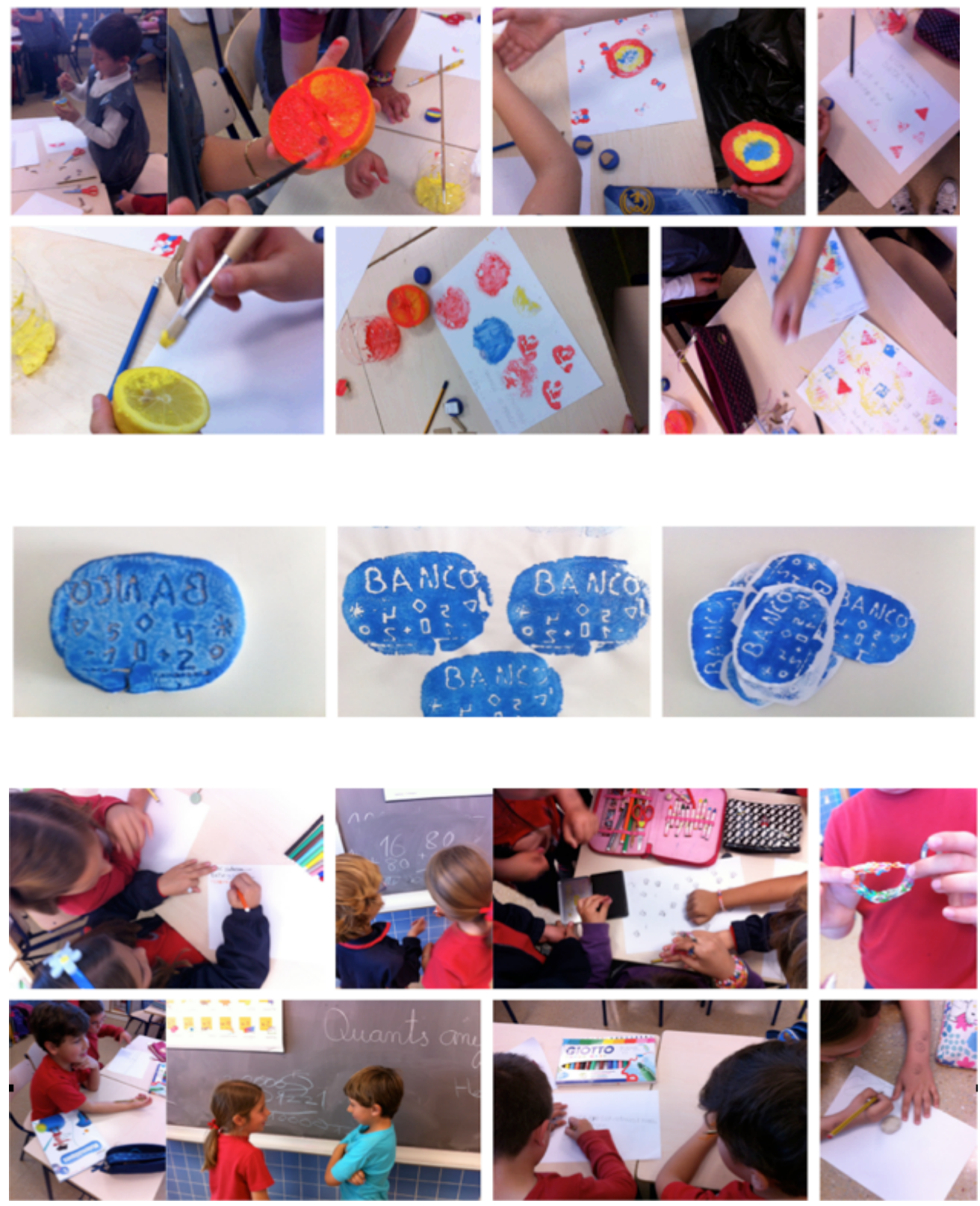

Figura 4. "Estampamos y Banco de aprendizaje" Montaje fotográfico de Oihana Tárrega. 
La quinta y última semana, la dedicamos a la elaboración de un museo en 3D, en este momento ya tenían suficiente información tanto visual como gráfica de todo tipo de espacios artísticos y elementos que podemos encontrar dentro de ellos, así como también el personal, obras de arte o esculturas.
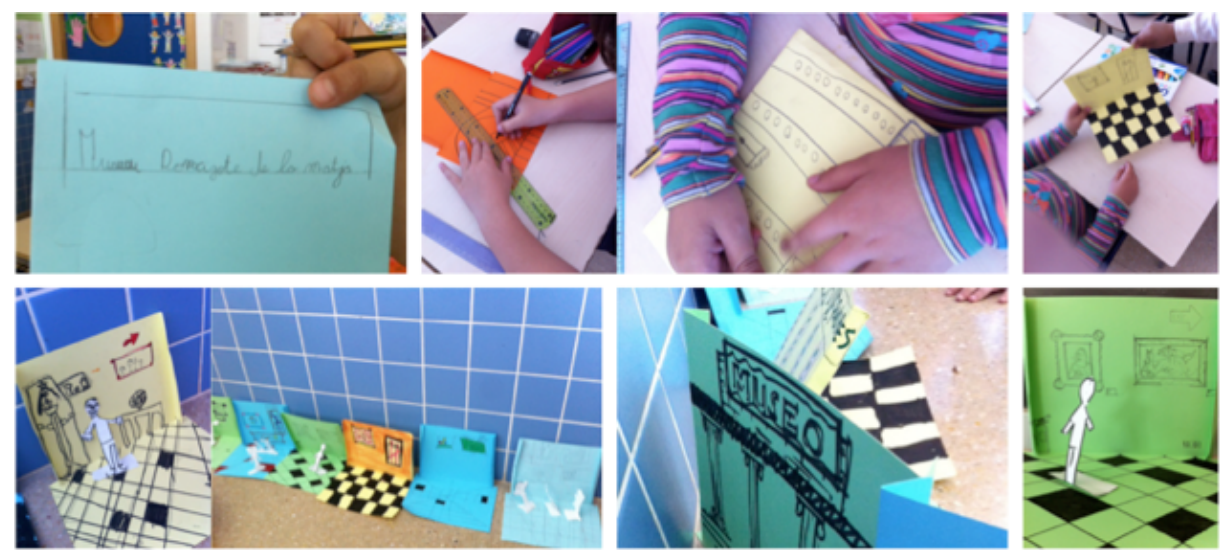

Figura 5. "Museo en 3D". Montaje fotográfico de Oihana Tárrega.

Después de esto y como final del proyecto, se generó una tabla con los nombres y actividades realizadas a lo largo de este tiempo. Cada alumno/a evaluó el resultado del trabajo realizado por los docentes colocando un punto rojo en aquellas actividades que no le habían gustado y un punto verde en aquellas que si. De esta manera eran partícipes tanto de las actividades como de su evaluación, nos ayudaron a mejorar aquellos aspectos en los que se había fallado y continuar con los que funcionaron. Por último, los maestros/as realizaron una evaluación final teniendo en cuenta los trabajos realizados, su participación y comportamiento entre otros factores.

\subsubsection{Recursos personales y materiales}

Los recursos personales serán los alumnos/as y los docentes. En cuestión de materiales los clasificamos en varios apartados: audiovisual, reciclado, peligroso, orgánico y de experimentación. Dentro de la sección audiovisual utilizaremos una presentación de Power Point para introducir la actividad, páginas web de diferentes museos, imágenes de cuadros de interés en la historia del arte, así como material de audio con música tradicional de la provincia.

Reciclaremos todo tipo de envases y botellas de plástico para colocar la pintura, tapones para realizar sellos, cartón en el que dibujar y recortar, rollos de papel higiénico y de cocina para hacer formas, estampar, etc.. Como objeto peligroso necesitaremos un cuchillo para cortar las frutas y verduras, que solo manipulará un docente y se mantendrá en todo momento alejado de los alumnos/as. También necesitaremos frutas y verduras como limón, berenjena, o manzana, podemos aprovechar aquellas que han madurado en exceso. 
Además utilizaremos otros elementos de uso común en el aula como: lápiz, goma, hojas de papel, cartulinas, tijeras, plastilina, rotuladores y pegamento, libros de texto. El único medio de transporte empleado será el autobús para el traslado del Colegio al Espacio de Arte Contemporáneo de Castellón, ubicado en la misma ciudad, a escasos 15 minutos.

\subsubsection{Cronograma}

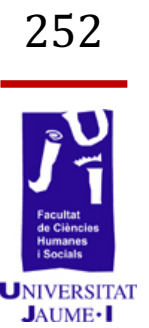

\begin{tabular}{|c|c|c|}
\hline Semanas & Actividad & Duración \\
\hline Semana 1 (3 al 7 de Marzo) & Visitante y artista & 50 minutos/sesión \\
\hline Semana 2 (11 Y 12 de Marzo) & Salimos del Colegio & 30 minutos/sesión \\
\hline Semana $3(17$ al 21 de Marzo) & Magdalena & 50 minutos/sesión \\
\hline Semana 4 (31 de Marzo al 16 de Abril) & Estampamos & 50 minutos/sesión \\
\hline
\end{tabular}

Figura 6.Cronograma de actividades

Cada una de las actividades ha sido titulada de una forma diferente, para que los alumnos/as identificasen sin problemas el contenido de las mismas, recordasen el trabajo realizado y les sirviera como motivación previa a la ejecución de las mismas.

\section{Resultados}

\subsection{Interés y relevancia del programa/material}

Actualmente nos encontramos cada vez con más ofertas educativas y la relación entre museo-escuela. Esta relación ha evolucionado del formato de visitas en las que los alumnado únicamente observaba las obras sin más información a actividades personalizadas y más elaboradas. Tras esta investigación, he podido comprobar que, hoy en día, los espacios de arte cuentan con gran cantidad de recursos didácticos para primaria. Lo cierto es que también existen proyectos muy creativos y pedagógicos relacionados con la Educación Primaria, en museos tales como el Prado en Madrid o Guggenheim de Bilbao en el que desde hace años existe un programa educativo interdisciplinar para reforzar las áreas del curriculum escolar de Educación Primaria, mediante actividades artísticas. Se llevan a cabo en los propios colegios, en los que un artista se desplaza hasta el mismo trabajando conjuntamente con los maestros/as. Sin embargo, en la oferta predominan los talleres en festividades como Navidad o épocas estivales, y por tanto son los padres/madres los que deben llevar a sus hijos/as.

\subsection{Resultados de la evaluación del programa/material}

Puesto que el EACC es un espacio público y gratuito al que todo el mundo tienen acceso, el primer paso por mi parte fue realizar una visita a 
la exposición. Sin obtener ningún tipo de información observe todas las obras expuestas, buscando a continuación por Internet datos de la exposición. Posteriormente, concerté una cita con el guía didáctico que muy amablemente me explicó cada una de las obras con todo tipo de detalles. Mi percepción de lo que veía cambió por completo, puesto que en un principio me parecía imposible explicarlo a niños/as de no más de 8 años, pero tras sus comentarios comprendí mejor el significado de las obras expuestas. Tras un par de reuniones comenzamos a trabajar conjuntamente, concretando las obras que parecían más interesantes y aquellos conceptos que los alumnos/as eran capaces de captar en base a sus conocimientos previos.

Establecimos un calendario de reuniones con intercambios de opiniones sobre como abordar la actividad. Es importante destacar que en los primeros encuentros se concretó que no era necesario hacer actividades previas a la salida directamente relacionadas con la exposición, sino que era preferible que se encontraran con ella. Consideramos interesante que el alumnado no tuviera ideas erróneamente preconcebidas, sino que fuera todo novedoso. En el aula hablamos de los museos, intercambiamos experiencias, conocimos visualmente algunos ejemplos, entramos en páginas web, observamos los diferentes tipos de exposiciones y obras que podemos encontrarnos, buscamos autores del momento y otros más antiguos, y conocimos las diferentes técnicas pictóricas. Es decir, creamos nuestro propio «museo« en el aula. De esta manera introdujimos los primeros conceptos y conseguimos estimular el interés por conocer un espacio nuevo pero manteniendo un elemento sorpresa, ya que los alumnos/as no sabían lo que allí les esperaba.

Durante la visita además de aprender disfrutaron mucho, por lo que después de la misma, consideramos oportuno seguir hablando sobre ésta, ya que era fundamental para afianzar lo que allí habían visto. Para ello, realizamos diversas actividades en el aula, centradas en una de las obras que consideré más interesante para trabajar, «El Banco del Tiempo» (Time/ Bank).

La exposición de arte contemporáneo «7.000.000.000» del EACC resultó ser compleja, ya que incluso un adulto tenía dificultades para comprender todos los significados del material allí expuesto sin ninguna guía. Esto venía a presagiar las dificultades que podríamos encontrarnos al trabajarla con los alumnos/as. Pero no fue así, la colaboración del guía didáctico ayudo a conseguir que llegasen a comprenderlo extrayendo de cada obra los conceptos y relacionarlos con lo que ellos conocen. De esta forma, no era necesario explicar la obra tal cual, sino adaptarla manteniendo su esencia. El responsable del gabinete didáctico controló en todo momento las actividades y todos los alumnos/as participaron, hablaron, rieron y saltaron, siendo todas las visitas muy dinámicas. Otro de los problemas que surge en este espacio es su capacidad de cambio, cada trimestre aproximadamente se renueva la exposición y con ellas el espacio interior, lo que impide programar visitas con antelación. Sin embargo las posibilidades de un espacio sin una exposición permanente son mayores, 
ya que permiten amoldar los recursos a las obras y a los visitantes. Otorgan una flexibilidad que en otro lugar no se daría.

\section{Discusión y Conclusiones}

\subsection{Comentarios críticos de las limitaciones y exposición de alternativas}

El colegio cuenta con una sala de usos múltiples, de grandes dimensiones, orientada hacia la montaña, con mesas de dibujo agrupadas, taburetes y dos fregaderos. Es un espacio diseñado para la realización de actividades plásticas en las que si se ensucia el lugar de trabajo existe la posibilidad de limpiar con agua, favoreciendo el buen mantenimiento de las instalaciones. Además de ello, factores como el cambio de aula, ayudan a que los alumnos/as muestren mayor interés por las actividades a realizar, ya que sustituyen actividades rutinarias y asocian este espacio a otro tipo de tareas. También influyen otros factores que favorecen el interés por trabajar con este espacio como la iluminación y las mesas de trabajo.

A pesar de ello, actualmente en esta aula se imparte el área de religión o su alternativa, por lo que no suele estar libre como norma general. El espacio destinado a realizar las actividades plásticas era el aula habitual, por lo que se hacía necesario la modificación de mesas y sillas para trabajar en grupo. Unos minutos antes de finalizar la clase, se dedicaban 5 minutos para organizarla de nuevo. Así mismo, cada alumno/a era responsable de dejar los materiales empleados en su lugar correspondiente.

Los materiales de los alumnos/as para Educación Plástica de libre acceso eran: lápices, goma y colores. Los rotuladores, plastilina, tijeras y pegamento estaban guardados en un armario, nuevos e intactos. Como se comentaba en uno de los puntos anteriores, el proyecto se trabajó tanto en primer curso de primaria como en segundo, además cada uno de ellos se divide en dos grupos, A y B. Los tutores de las diferentes clases, eran los encargados de seleccionar y pedir el material necesario para todo el curso escolar. Por este motivo, el material en todas las clases no era el mismo, lo que dificultaba el realizar las mismas actividades con todos los alumnos/as.

Para llevar a cabo el proyecto eran necesarios materiales concretos, por lo que le propuse al tutor-supervisor que los alumnos/as trajeran de casa una fruta o verdura y guardasen material reciclado como tapones de botellas, rollos de papel higiénico y de cocina. El tutor del centro no consideró oportuno pedir a los padres/madres material ni colaboración, porque estamos en un momento económico difícil, algo que considero hubiera sido muy positivo para las actividades, ya que familias e hijos/as tenían la posibilidad de colaborar en la actividad y les hacíamos partícipes. Además, entre todos podríamos haber conseguido mucho material y hubiéramos reciclado, tomando conciencia de lo necesario que resulta. Finalmente y debido al interés en llevar a cabo las actividades del recurso didáctico, me encargué de conseguir el material. Además, empleamos todo lo disponible en el aula, comentado anteriormente. 
Para terminar, y en relación a la coordinación entre todas las clases y los tiempos, comentar que la actividad la realizábamos en todo primer ciclo, con dos grupos por curso. Esto conllevaba una complicada organización de las 4 clases con sus respectivas sesiones, ya que los alumnos/as de primero tenían dos a la semana y los de segundo solamente una. El tiempo tampoco jugaba a nuestro favor, por lo que se adaptaron las actividades a los 50 minutos de duración.

\subsection{Resultados y evaluación de su aplicación}

La evaluación del alumnado ha sido cualitativa y cuantitativa. Se han tenido en cuenta los trabajos y el comportamiento en una evaluación inicial, otra procesual y otra final, como se muestra en la tabla de evaluación docente. Además, se ha realizado una autoevaluación del proyecto, en la que han participado los alumnos/as dando su opinión positiva o negativa a las actividades llevadas a cabo. Por otro lado, se ha seleccionado una muestra de las notas de los 25 alumnos/as de una de las cuatro clases, antes de la experiencia y después de ella.

El análisis de los resultados muestra que el recurso didáctico ha funcionado, ya que los datos obtenidos han sido muy positivos. Lógicamente como se comenta en apartados anteriores, no todo es fruto de las actividades realizadas en Educación Plástica, sino que influyen otros factores como el aumento del personal docente en el aula, lo que mejora positivamente el control y atención de los alumnos/as. Además de ello otro factor que influye es el desarrollo natural de los niños/as, puesto que en el primer trimestre están adaptándose a términos y actividades novedosas y a medida que avanza el curso van progresando, aprendiendo y mejorando.

En la escuela, los docentes no mostraron demasiado entusiasmo con la salida a un espacio de arte, incluso me agradecieron que fuese la encargada, ya que conlleva un aumento de las responsabilidades. Aún así fueron invitados a las reuniones realizadas con el guía didáctico y a visitar la exposición. Personalmente me involucré e hice una primera visita en la que simplemente observé las obras expuestas y el espacio. Me sorprendió gratamente la amabilidad y dedicación que el Espai d'Art Contemporani de Castellón estaba mostrando hacia los que iban a ser sus visitantes. Conseguimos que fuera una visita guiada a la carta, diseñada para ellos, donde EACC y escuela estábamos unidos para que todo saliera bien.

Como conclusión señalar que en mi opinión está ha sido una gran experiencia, en la que aprendimos unos de otros. Las dificultades y los inconvenientes iniciales, dieron paso a un trabajo cuidado y estudiado que conllevó que todas las actividades salieran mejor de lo esperado. Los alumnos/as asombraron por su iniciativa en el trabajo y creatividad en sus composiciones, ayudados sin duda porque la mayoría de ellos no estaban acostumbrados a trabajar con pinturas ni pinceles, algo que les sorprendió y agradó al mismo tiempo. 
Para terminar, espero que mucha gente ponga la misma ilusión, ganas y esfuerzo en realizar proyectos de Educación Plástica porque entre todos podemos conseguir que cobre la misma importancia que las demás, pues como se ha mencionado y demostrado ayuda al desarrollo personal e intelectual de nuestros alumnos/as.

\begin{tabular}{|c|c|c|c|}
\hline Área & Mejora & $\begin{array}{c}\text { Se } \\
\text { mantiene }\end{array}$ & Empeora \\
\hline Matemáticas & 12 & 10 & 3 \\
\hline Valenciano & 13 & 8 & 4 \\
\hline C. del medio & 10 & 12 & 3 \\
\hline Plástica & 18 & 7 & 0 \\
\hline
\end{tabular}
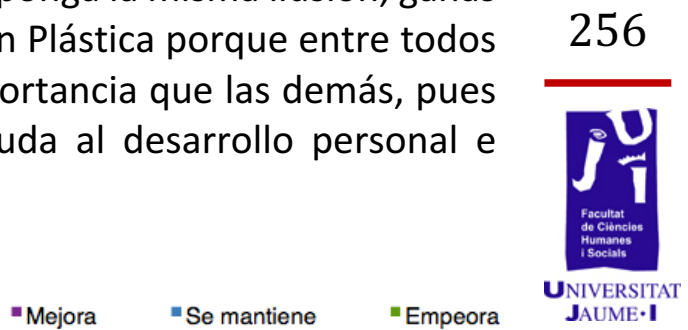

Figura 7. Evaluación de las actividades

\section{Bibliografía}

AcAso, M. (2009): La Educación artística no son manualidades: Nuevas prácticas en la enseñanza de las artes y la cultura visual, La Catarata, Madrid.

Barbe-Gall, F. (2009): Cómo hablar de arte, Nerea, Donostia-San Sebastián.

Calaf, R. y O. Fontal. (2010): Cómo enseñar arte en la escuela, Sintesis, Madrid.

Camareno-Izquierdo, C., et. al. (2009): "Generating Emotions through Cultural Activities in Museums «. International Review on Public and non Profit Marketing. 6, 2. 151-165.

DeWEY, J. (1934): Art As Experience, Balch and Company, Nueva York.

EISNER, E. (1995): Educar la visión artística, Paidós Educador, Barcelona.

FERNÁNDEZ, O. (2007): «El lugar de la palabra. El departamento de investigación y educación del Museo Patio Herreriano», en Fernández, $\mathrm{O}$ y V. del Río. (ed.): Estrategias críticas para una práctica educativa en el arte contemporáneo (pp. 19-24). Museo Patio Herreriano, Valladolid.

KaUfman. I. (1965): "Report of the Commission on Art Education», National Art Education Association, 25.

LOWENFELD, V. y LAMBeRt BRItTAIN, W. (1980): Desarrollo de la capacidad creadora. Buenos Aires, Kapelusz.

LUCAS,K.B. (2000) «One Teacher's Agenda for a Class Visit to an Interactive Science Center», Science Education, 84. 524-544.

Machuca Carvajal, G. y A. Villareal Mesa (2012): Arte contemporáneo en la escuela, Guía didáctica complementaria para profesores de Artes Visuales 
de enseñanza media. Obtenido el 22 de abril de 2014 http://galeriagm.cultura.gob.cl/uploads/educacion_archivo/cb016e6b92. pdf

Marín-Viadel,R. (2003): Didáctica de la Educación Artística para Primaria. Pearson Educación, Madrid.

SANChez Mora, M. (2013): "La relación Museo-Escuela: tres décadas de investigación educativa». El Museo y la Escuela. Conversaciones de complemento, 15-21.

StORKSDIECK, M. (2006): Field Trips in Environmental Education. Berlín: Berliner Wissens- chafts- Verlag.

Trilla, J. y otros (2003): La educación fuera de la escuela. Ámbitos no formales y educación social. Editorial Ariel, Barcelona.

\section{Webgrafía}

Actividades niños y familias (2014). Obtenido el 10 de marzo de 2014 de http://www.guggenheim-bilbao.es/actividades/perfil/ninos-y-familias/.

Alumnos de primaria se acercan al arte contemporáneo en Huarte (2014). Obtenido el 2 de marzo de 2014

http://www.diariodenavarra.es/noticias/navarra/pamplona comarca/20 14/02/26/alumnos primaria acercan arte contemporaneo huarte 149 265_1002.html.

BOE (2013). Obtenido el 12 de febrero de 2014 de http://www.boe.es/boe/dias/2013/12/10/pdfs/BOE-A-2013-12886.pdf

Decreto 111/2007 (2007) . Obtenido el 12 de abril de 2014 de http://www.docv.gva.es/datos/2007/07/24/pdf/2007_9730.pdf.

Diego Velazquez (2010). Obtenido el 10 de marzo de 2014 de https://recursosep.files.wordpress.com/2010/11/unidad-1g.pdf

Educación reduce Plástica para ampliar horas de Lengua y Mates (2014). Obtenido el 10 de febrero de 2014 de http://ccaa.elpais.com/ccaa/2014/01/21/madrid/1390324545_217503.ht $\underline{\mathrm{ml}}$.

El arte contemporáneo irrumpe en las aulas (2013). Obtenido el 15 de abril de 2014 de http://www.mustangartgallery.com/el-arte-contemporaneoirrumpe-en-las-aulas.

Entrevista con María Acaso (2014). Obtenido el 24 de abril de 2014 de http://centredart.bcn.cat/es/content/entrevista-con-mar\%C3\%ADa-acaso Formación profesorado (2014). Obtenido el 2 de abril de 2014 de https://www.museodelprado.es/educacion/formacion-para-elprofesorado/ Materiales Escolares (2009). Obtenido el 15 de febrero de 2014 de 
http://www.edelvives.com/escolar/primaria/pixepolis

Programa d'immersió lingüística (2010). Obtenido el 15 de abril de 2014

https://sites.google.com/a/ceiptombatossals.edu.gva.es/inici/el-

centre/programes.

Programa Traid 4ㅇ de Primaria. (2013). Obtenido el 25 de abril de 2014 de http://www.tradid.es/Programa-Tradid-4-de-primaria.

Real Decreto 1513/2006 (2006). Obtenido el 12 de febrero de 2014 de https://www.boe.es/buscar/doc.php?id=BOE-A-2006-21409

«7.000.000.000» Un projecte comissariat per David Arlandis i Javier Marroquí. (2014). Obtenido el 5 de abril de 2014 de http://www.eacc.es/blog/7-000-000-000-expo/

Visita taller (2009). Obtenido el 18 de marzo de 2014 de http://www.museothyssen.org/thyssen/visita_privada_taller. 